\title{
Analysis of biological neuron and training of an artificial neuron for a neural processor construct
}

\begin{abstract}
The basic idea behind a neural processor is to simulate lots of densely interconnected artificial neuron inside a computer so you can get it to learn things, recognize patterns, and make decisions in a humanlike way. The best thing about a neuron processor is that you don't have to program it to learn explicitly: it learns all by itself, just like a brain. In this paper analysis of biological neurons are made to make a successful artificial neural processor that can be used in robots for analyzing the problem in detail by itself. Automatic training of neuron network is also been performed including data analysis with an example.
\end{abstract}

Keywords: neuron, neural processor, neural network, data analysis, specific structures
Volume 4 Issue 6 - 2018

\section{Manu Mitra}

Electrical Engineering Department, University of Bridgeport, USA

Correspondence: Manu Mitra, Electrical Engineering Department, University of Bridgeport , 126 Park Avenue, Bridgeport, CT - 06604, USA,

Emailmmetra@my.bridgeport.edu

\section{Introduction}

The brain is an assembly of interconnected neurons. Neurons attain in a range of shapes and sizes, contingent on their capacity and specific structures. Nevertheless, in common, all neurons work in the comparative way and take after each other. Likewise, neurons of animals look similar like the neurons of people. The normal number of neurons in human cerebrum is around 100 billion. Regardless of whether human is the most brilliant animal on the planet, some fascinating insights about neurons in people and different creatures, demonstrated that there are creatures that may have a more noteworthy measure of neurons than the human. A fascinating certainty about octopus is that normal number of neurons in an octopus brain is around 300billion.

Every neuron is a cell that utilizes biochemical responses to get process and transmit data. A neuron's dendrite tree is associated with a thousand neighboring neurons. When one of those neurons fires, a positive or negative charge is caught by one of the dendrites. The qualities of all the caught charges are included through the procedures of spatial and worldly summation. Spatial summation happens when a few powerless signs are changed over into a solitary expansive one, while fleeting summation changes over a quick arrangement of feeble heartbeats from one source into one huge flag. The total info is then passed to the soma (cell body). The soma and the encased core don't assume a critical part in the preparing of approaching and active information. Their essential capacity is to play out the consistent support required to keep the neuron practical. The piece of the soma that concerns itself with the flag is the axon hillock.

Electrical driving forces from the bot enter the bunch of neurons, and reactions from the cells are transformed into charges for the device. Cells can shape new associations, making the framework a learning machine. For the most part neuron control causes the robot to keep away from dividers. However that snag shirking frequently indicates clear change after some time, exhibiting how systems of neurons can allow basic assuming out how to the machines (Figure $1 \& 2) .{ }^{1,2}$

\section{Analyzing biological neuron for an artificial neuron processor construct}

Below are the graphs were taken for analyzing a biological neuron. ${ }^{3,4}$ Permission was granted by Prof. Eric Newman to use graphs in this paper generated by Meta Neuron. ${ }^{5}$

\section{a. Resting membrane potential}

Membrane potential characteristics (Figure 3)

Sodium

Concentration out $(\mathrm{mM})=120$

Concentration in $(\mathrm{mM})=16.4859$

$\mathrm{Na}+$ equilib potential $(\mathrm{mV})=50.00$

Potassium

Concentration out $(\mathrm{mM})=$ Range

Concentration in $(\mathrm{mM})=63.7834$

$\mathrm{K}+$ equilib potential $(\mathrm{mV})=11.58$

Relative Membrane Permeability

$\mathrm{Na}+$ permeability $=1$

$\mathrm{K}+$ permeability $=65$

Membrane Potential

Potential $(\mathrm{mV})=11.93$

\section{b. Membrane time constant}

Membrane time constant characteristics (Figure 4)

Membrane resistance

$\mathrm{K} \Omega \mathrm{cm}^{2}=10$

Stimulus

Delay $(\mathrm{ms})=2$

Width $(\mathrm{ms})=1$

Amplitude $(\mu \mathrm{A})=10$

Period $(\mathrm{ms})=3$

Number of stimuli=Range

Sweep duration $(\mathrm{ms})=40$

Range value 1 to 5 


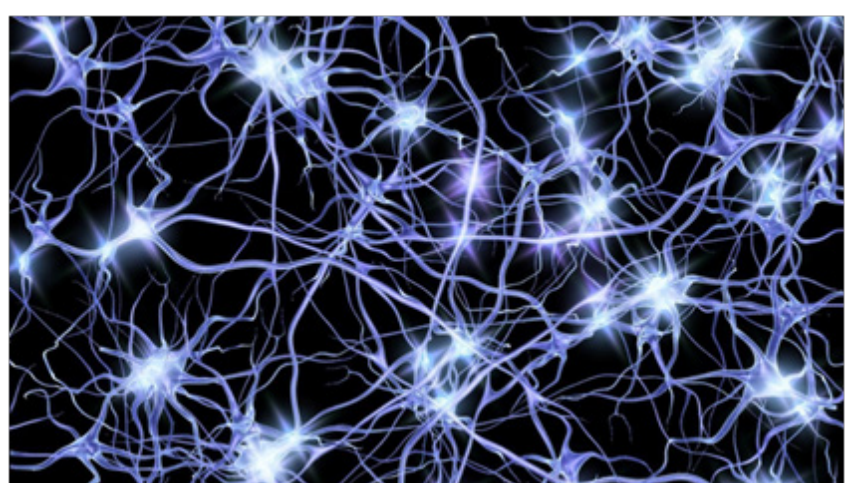

Figure I Depicts multiple biological natural neuron connected to each other. ${ }^{3}$

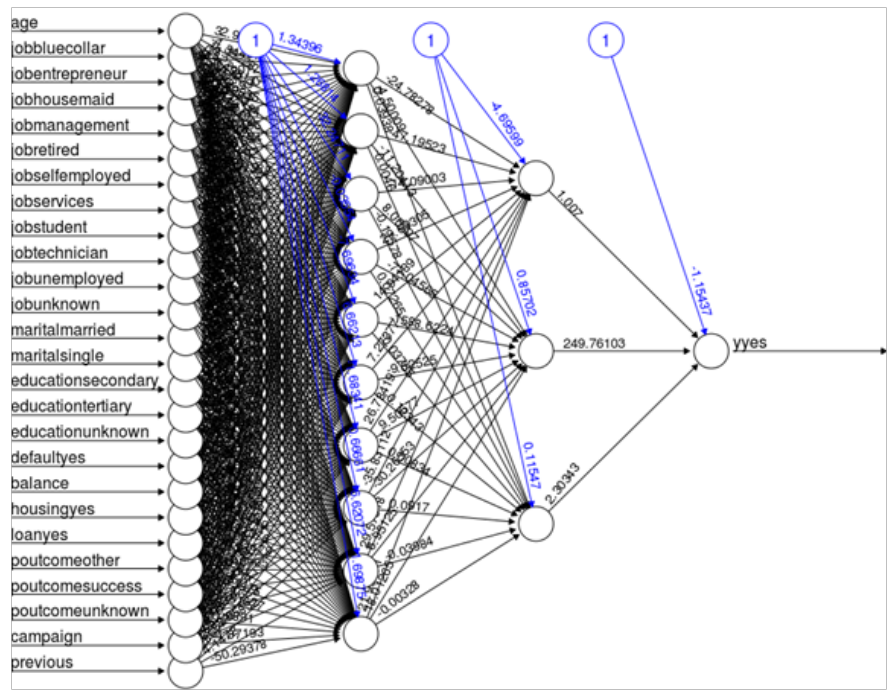

Figure 2 Depicts artificial neuron (Neural Network) connected to each other with hidden layers that cannot be seen explicitly in biological neuron. ${ }^{4}$

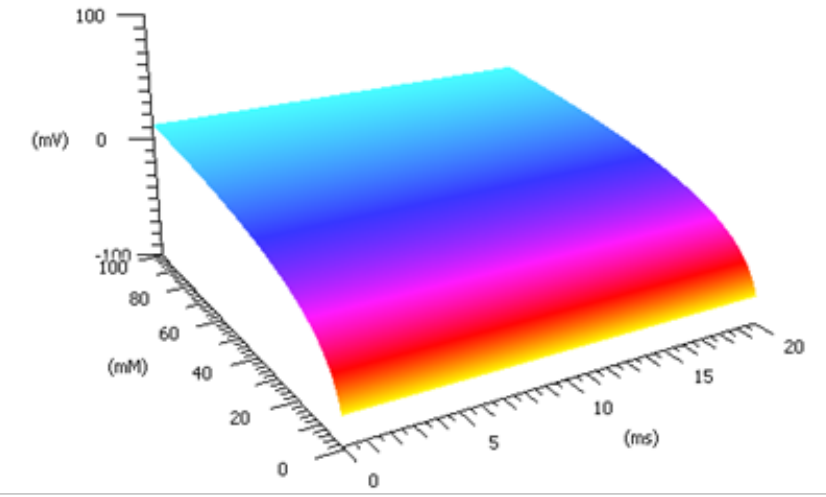

Figure 3 Depicts 3D graph of resting membrane potential.

\section{c. Membrane length constant}

Membrane length constant characteristics (Figure 5)

Dendrite/Axon Properties

Membrane Resistance $\left(\mathrm{k} \Omega-\mathrm{cm}^{2}\right)=5$
Internal Resistivity $(\Omega-\mathrm{cm})=$ Range

Diameter $(\mu \mathrm{m})=0.1$

Membrane capacitance $=1 \mu \mathrm{F} / \mathrm{cm} 2$

Stimulus

Synaptic Potential

Delay $(\mathrm{ms})=1$

Width $(\mathrm{ms})=50$

Amplitude $(\mu \mathrm{A})=100$

Potential vs Distance

Enabled

Time $(\mathrm{ms})=50$

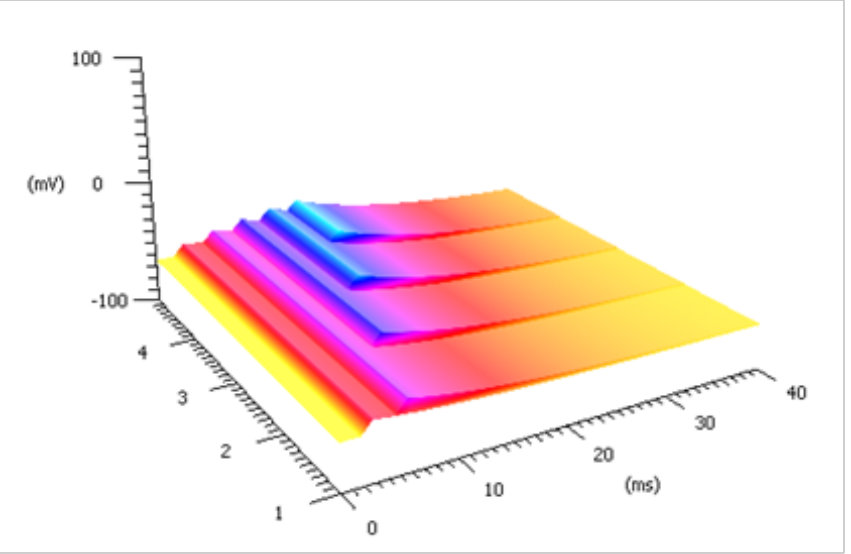

Figure 4 Depicts 3D graph of membrane time constant.

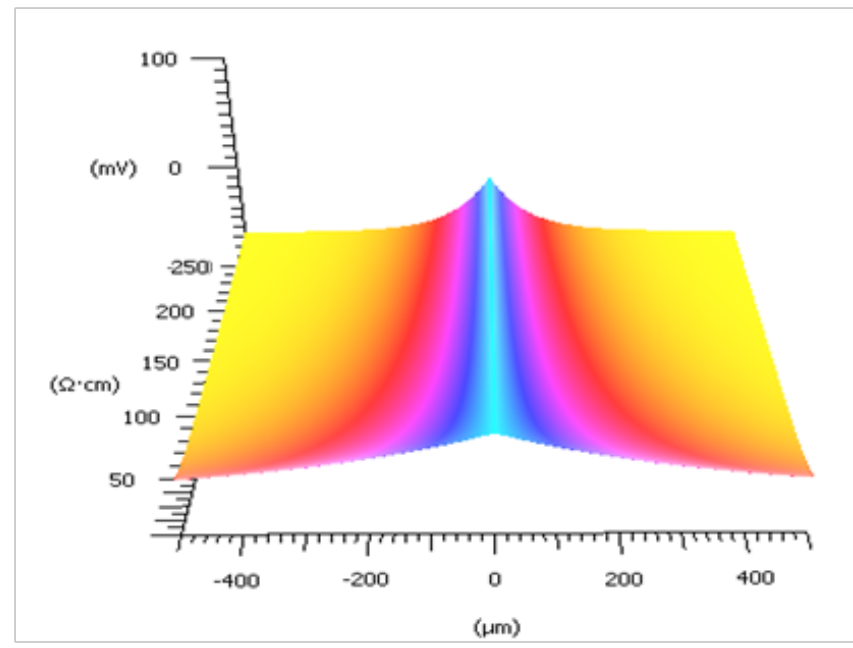

Figure 5 Depicts 3D graph of membrane length constant.

\section{d. Axon action potential}

Axon action potential characteristics (Figure 6)

Membrane parameters

$\mathrm{Na}+$ equilib potential $(\mathrm{mV})=50$

$\mathrm{gNa} \max (\mathrm{mS} / \mathrm{cm} 2)=260$ 
$\mathrm{K}+$ equilib potential $(\mathrm{mV})=-77$

$\operatorname{gK} \max (\mathrm{mS} / \mathrm{cm} 2)=70$

Membrane leakage

Reversal potential $(\mathrm{mV})=-55$

gLeak $(\mathrm{mS} / \mathrm{cm} 2)=0.6$

Conductance and Currents

Ionic currents

Holding Current

Holding $(\mu \mathrm{A})=$ Range

Stimulus

Delay $(\mathrm{ms})=0.5$

Width $(\mathrm{ms})=0.1$

Amplitude $(\mu \mathrm{A})=65$

Temperature 18 degree Centigrade

Range value -5 to 15

Sweep duration $(\mathrm{ms})=5$

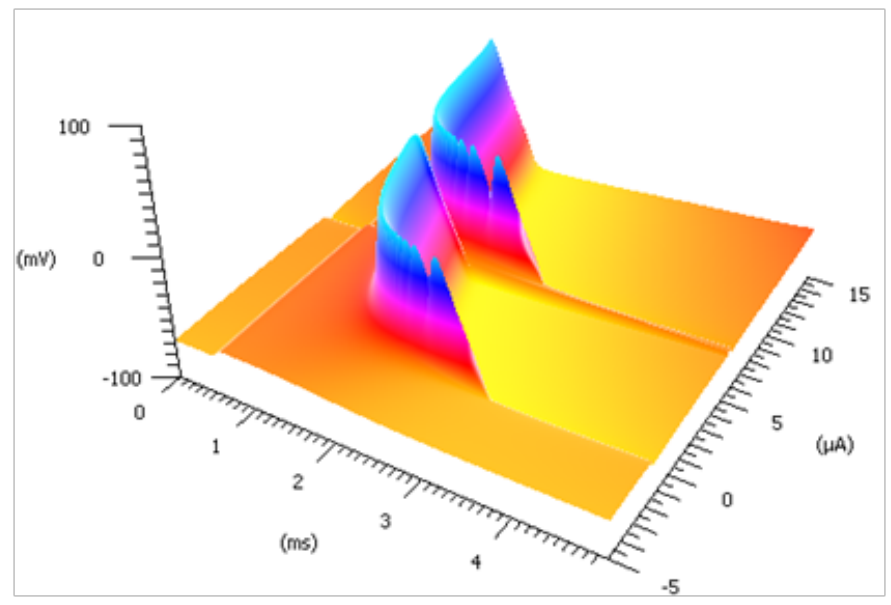

Figure 5 Depicts 3D graph of membrane length constant.

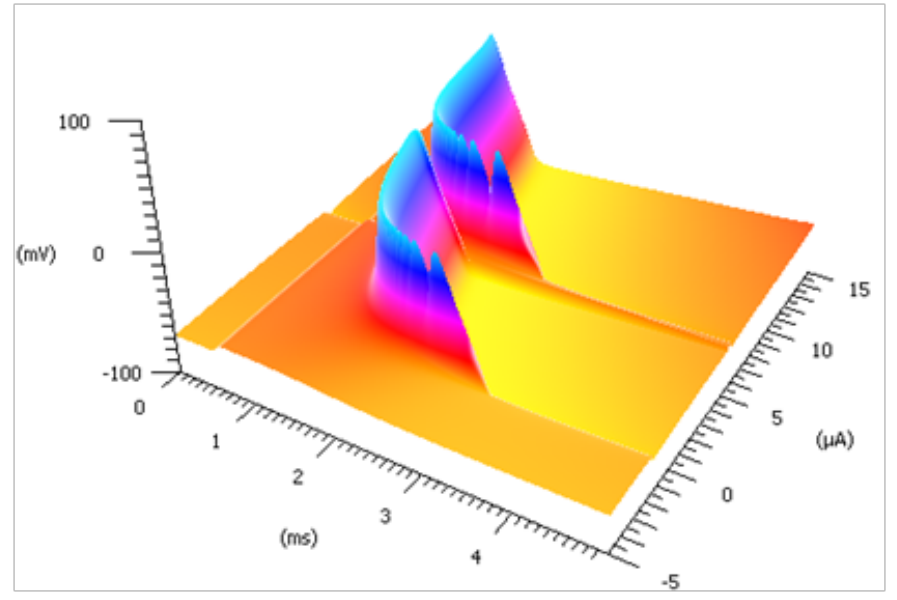

Figure 6 Depicts 3D graph of axon action potential.

\section{e. Axon voltage clamp}

Axon action potential characteristics (Figure 7)

Membrane Parameters

$\mathrm{Na}+$ equilib potential $(\mathrm{mV})=50$

$\mathrm{gNa} \max (\mathrm{mS} / \mathrm{cm} 2)=260$

$\mathrm{K}+$ equilib potential $(\mathrm{mV})=-77$

$\mathrm{gK} \max (\mathrm{mS} / \mathrm{cm} 2)=70$

Membrane Leakage

Reversal potential $(\mathrm{mV})=-55$

gLeak $(\mathrm{mS} / \mathrm{cm} 2)=0.6$

Holding potential

Holding $(\mathrm{mV})=$ Range

Stimulus

Delay $(\mathrm{ms})=1$

Width $(\mathrm{ms})=4$

Amplitude $(\mu \mathrm{A})=-5$

Temperature 18 degree Centigrade

Range value -75 to -30

Sweep duration $(\mathrm{ms})=8$

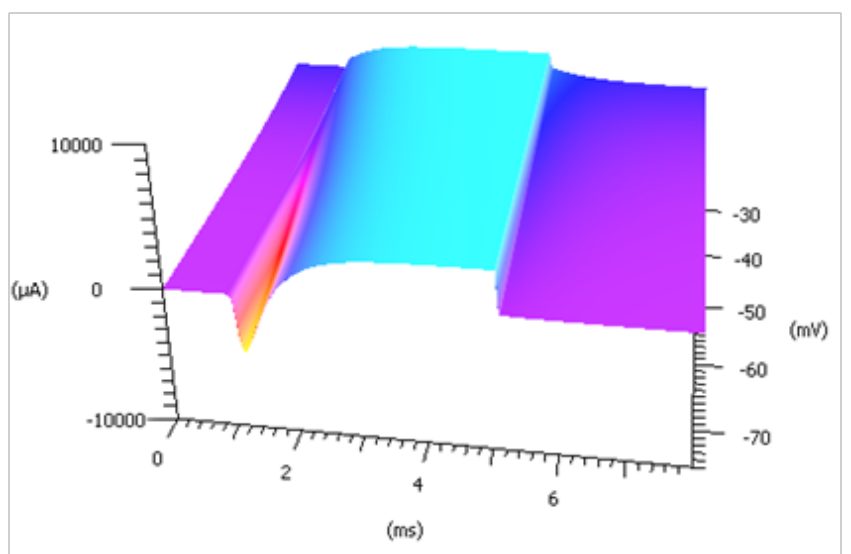

Figure 7 Depicts 3D graph of axon voltage clamp.

\section{f. Synaptic potential and current}

Synaptic potential and current characteristics (Figure 8)

Ion permeability

$\mathrm{Na}+$ permeability $=1.2$

$\mathrm{K}+$ permeability $=1$

Synapse type

Fast excitatory synapse

Holding Current

Holding $(\mu \mathrm{A})=$ Range 


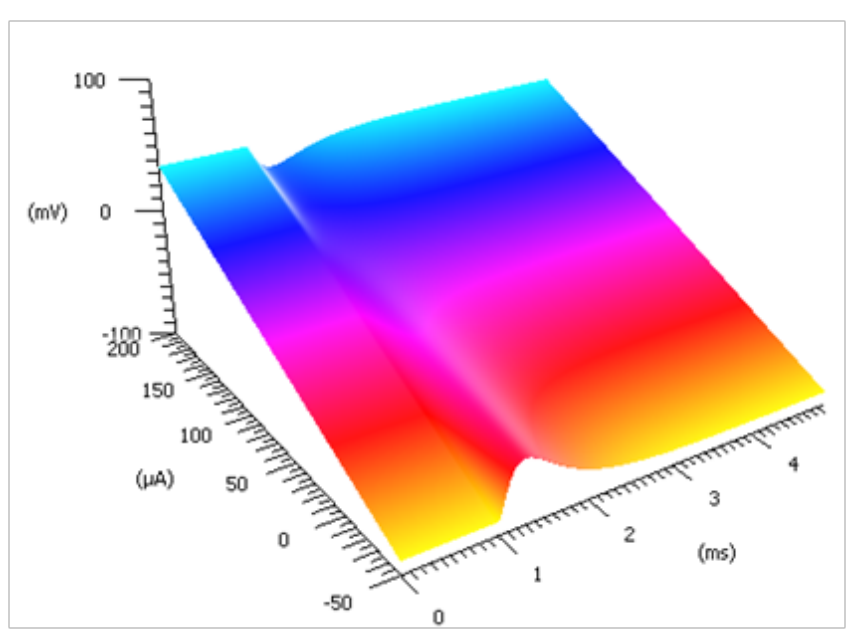

Figure 8 Depicts 3D graphs of synaptic potential and current.

\section{Observations of biological neuron graphs}

As per the above graphs when designing a neuron processor these specifications are to be considered such Resting Membrane Potential, Membrane Time Constant, Membrane length Constant, Axon action potential, Axon voltage clamp, synaptic potential and current. All the graphs were plotted based on voltage and/or current and assigning range of currents $(\mu \mathrm{A})$ and voltage $(\mathrm{mV})$. Hence it's vital for any neural processor what amount of voltage and current is being delivered to get desired output. ${ }^{6}$

\section{Training of artificial neurons}

Training (Table 1\&2) of an artificial neuron network (Part-I) (Figure 9-12)

An experiment was performed for training of neurons.

Training of an artificial neuron network (Part-II) (Figure 13 \&14)

\begin{tabular}{|c|c|c|c|}
\hline \multicolumn{4}{|c|}{ Network training (100\%) } \\
\hline \multicolumn{4}{|c|}{ Net Model: Back-Prop (MLP Base 2-10-1) committee[0] } \\
\hline \multicolumn{4}{|c|}{ mesg: BPQuickPropOptimize } \\
\hline iters done: 78883 & born: 0 & killed: 0 & Stop! \\
\hline iter: $1729 / 2000$ & err: 0.036 & 96971 & $1 \mathrm{~ms}$ \\
\hline
\end{tabular}

Figure 9 Depicts training of a neuron network.

\section{Data analysis}

Data Analysis was performed on Neural Networks (Table 3) to distinguish between various species of geranium flowers Figure 15 This example demonstrates the use of both input categories (Regular/ Irregular, Herbaceous/Succulent) and output categories (the five geranium types) (Figure16). ${ }^{7}$

\section{Experimental data feed to neural networks}

Experimental data feed was performed to Neural Network and output data from a neuron was documented below (Table 4).

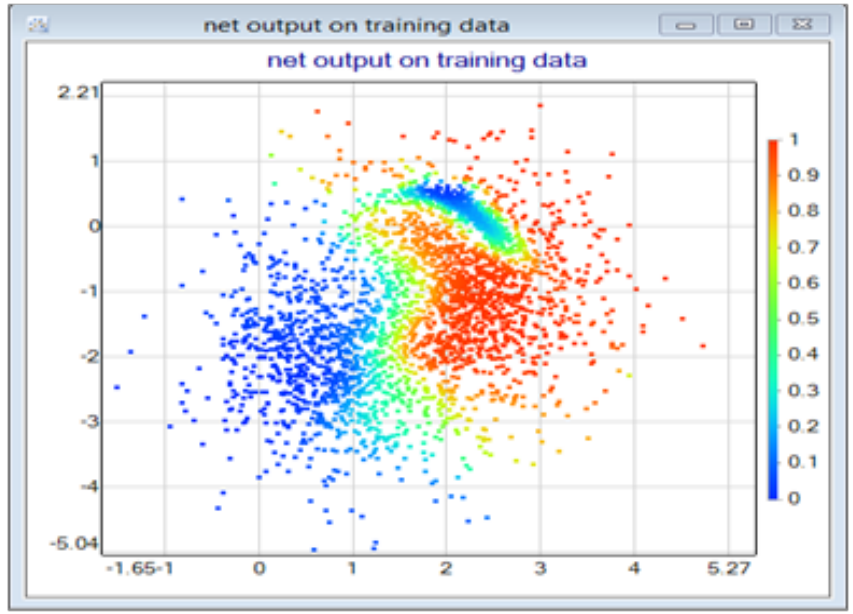

Figure 10 Depicts net outputs on training data.

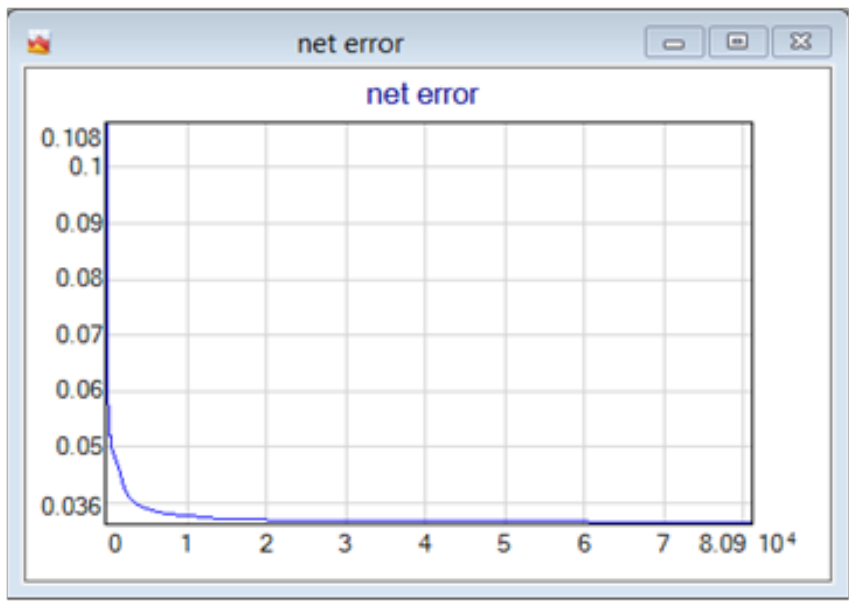

Figure II Depicts net error after training data.

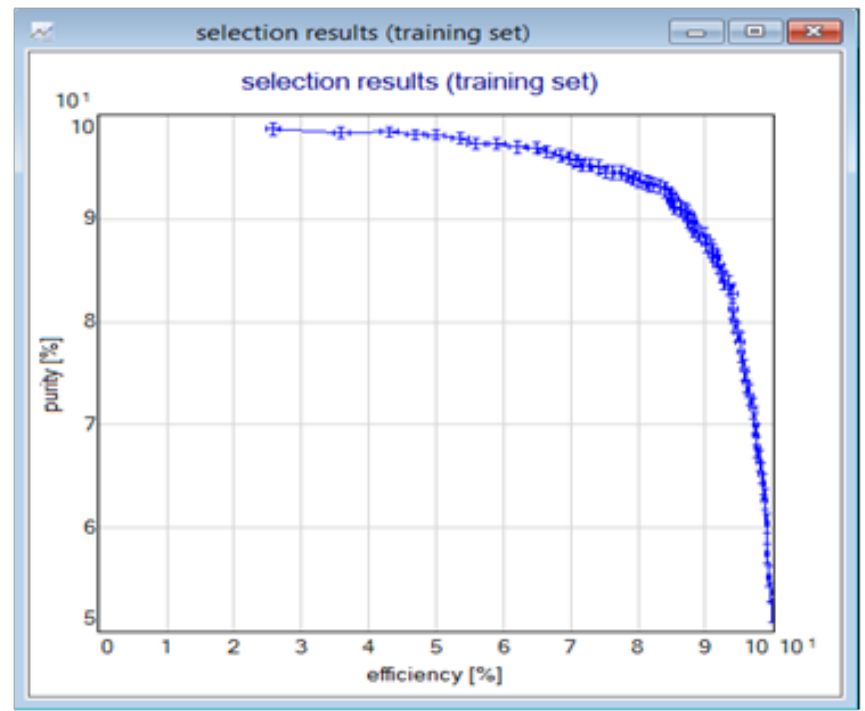

Figure 12 Depicts selection results (training set) efficiency vs purity. 


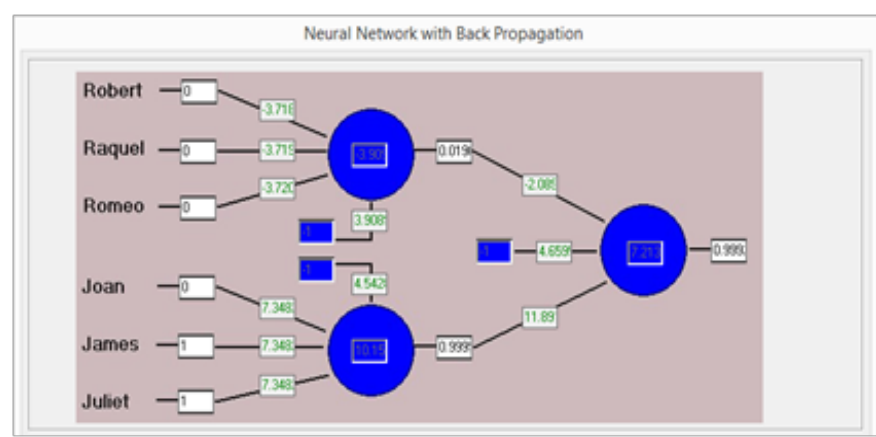

Figure 13 Depicts neural network training using back propagation.

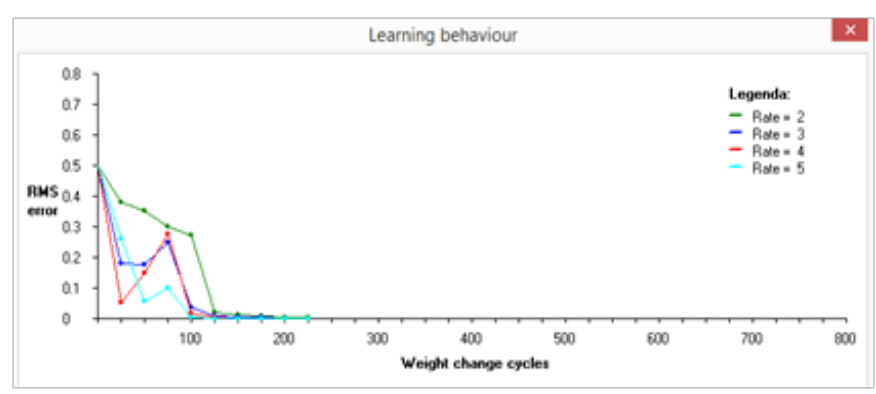

Figure I4 Depicts neural network learning behavior.

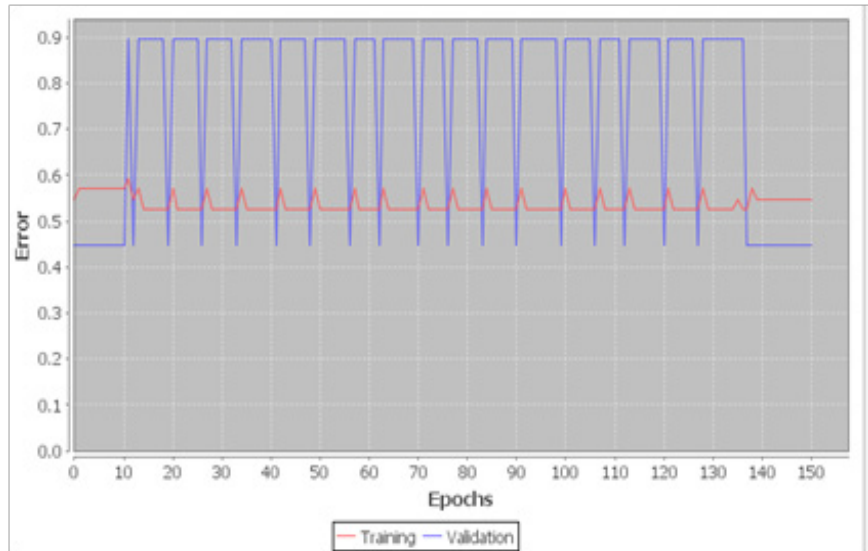

Figure 15 Depicts graphs epoch's vs error.

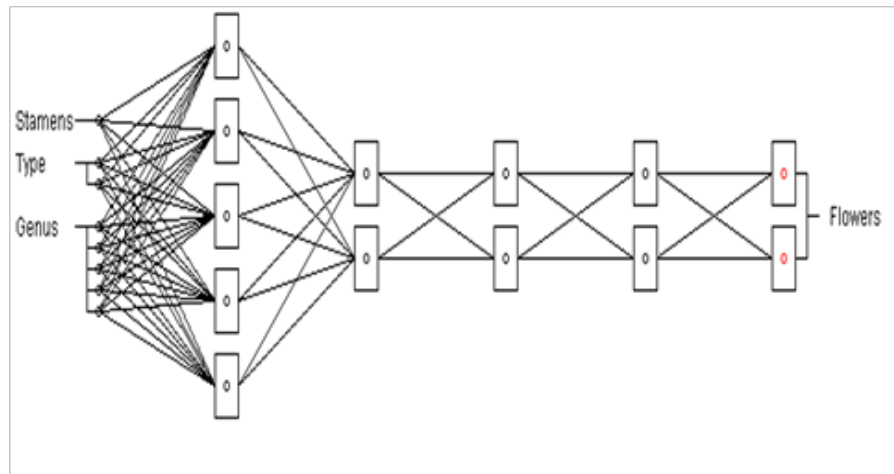

Figure 16 Depicts neuron set including hidden layers.

Table I Training results

\section{S \\ 5 \\ 5 \\ 5}

12

12

15

10

12

12

10

15

10

5

10

15

12

10

5

12

15

5

15

15

5

12

5

5

10

10

12

12

10

10

12

12

15

5

5

10

12

\begin{tabular}{|c|c|c|c|c|}
\hline tamens & Type & Genus & Flowers & Actual \\
\hline \multirow[t]{3}{*}{2} & Succulent & Pelargonium & Irregular & Regular \\
\hline & Succulent & Erodium & Regular & Regular \\
\hline & Succulent & Erodium & Regular & Regular \\
\hline \multirow[t]{2}{*}{2} & Herbaceous & Monsonia & Regular & Regular \\
\hline & Succulent & Erodium & Regular & Regular \\
\hline 5 & Herbaceous & Monsonia & Regular & Regular \\
\hline 0 & Herbaceous & Geranium & Regular & Regular \\
\hline 2 & Herbaceous & Monsonia & Regular & Regular \\
\hline 2 & Succulent & Sarcocaulon & Regular & Regular \\
\hline 0 & Herbaceous & Geranium & Regular & Regular \\
\hline 5 & Succulent & Pelargonium & Irregular & Regular \\
\hline \multirow[t]{2}{*}{0} & Succulent & Pelargonium & Irregular & Regular \\
\hline & Succulent & Pelargonium & Irregular & Regular \\
\hline 0 & Succulent & Geranium & Regular & Regular \\
\hline 5 & Succulent & Sarcocaulon & Regular & Regular \\
\hline 2 & Herbaceous & Monsonia & Regular & Regular \\
\hline \multirow[t]{2}{*}{0} & Succulent & Pelargonium & Irregular & Regular \\
\hline & Herbaceous & Pelargonium & Irregular & Regular \\
\hline 2 & Herbaceous & Monsonia & Regular & Regular \\
\hline \multirow[t]{2}{*}{5} & Herbaceous & Monsonia & Regular & Regular \\
\hline & Succulent & Erodium & Regular & Regular \\
\hline 5 & Succulent & Sarcocaulon & Regular & Regular \\
\hline \multirow[t]{2}{*}{5} & Herbaceous & Monsonia & Regular & Regular \\
\hline & Herbaceous & Pelargonium & Irregular & Regular \\
\hline \multirow[t]{3}{*}{2} & Succulent & Sarcocaulon & Regular & Regular \\
\hline & Herbaceous & Erodium & Regular & Regular \\
\hline & Herbaceous & Erodium & Regular & Regular \\
\hline 0 & Herbaceous & Geranium & Regular & Regular \\
\hline 0 & Succulent & Geranium & Regular & Regular \\
\hline 2 & Herbaceous & Monsonia & Regular & Regular \\
\hline 2 & Succulent & Pelargonium & Irregular & Regular \\
\hline 0 & Herbaceous & Geranium & Regular & Regular \\
\hline 0 & Herbaceous & Pelargonium & Irregular & Regular \\
\hline 2 & Succulent & Sarcocaulon & Regular & Regular \\
\hline 2 & Succulent & Sarcocaulon & Regular & Regular \\
\hline 5 & Herbaceous & Monsonia & Regular & Regular \\
\hline & Succulent & Erodium & Regular & Regular \\
\hline & Succulent & Erodium & Regular & Regular \\
\hline 0 & Succulent & Geranium & Regular & Regular \\
\hline 2 & Succulent & Sarcocaulon & Regular & Regular \\
\hline
\end{tabular}

Citation: Mitra M.Analysis of biological neuron and training of an artificial neuron for a neural processor construct. Int Rob Auto J. 20 I8;4(6):407-4I7. DOI: 10.15406/iratj.2018.04.00157 
Table Continued

\begin{tabular}{|c|c|c|c|c|}
\hline Stamens & Type & Genus & Flowers & Actual \\
\hline 15 & Herbaceous & Monsonia & Regular & Regular \\
\hline 10 & Herbaceous & Geranium & Regular & Regular \\
\hline 5 & Succulent & Erodium & Regular & Regular \\
\hline 10 & Herbaceous & Pelargonium & Irregular & Regular \\
\hline 5 & Herbaceous & Erodium & Regular & Regular \\
\hline 10 & Succulent & Geranium & Regular & Regular \\
\hline 12 & Herbaceous & Monsonia & Regular & Regular \\
\hline 10 & Herbaceous & Geranium & Regular & Regular \\
\hline 15 & Herbaceous & Monsonia & Regular & Regular \\
\hline 5 & Herbaceous & Pelargonium & Irregular & Regular \\
\hline 12 & Herbaceous & Monsonia & Regular & Regular \\
\hline 10 & Succulent & Pelargonium & Irregular & Regular \\
\hline 10 & Succulent & Geranium & Regular & Regular \\
\hline 15 & Succulent & Sarcocaulon & Regular & Regular \\
\hline 12 & Succulent & Sarcocaulon & Regular & Regular \\
\hline 15 & Succulent & Sarcocaulon & Regular & Regular \\
\hline 10 & Succulent & Geranium & Regular & Regular \\
\hline 5 & Succulent & Erodium & Regular & Regular \\
\hline 15 & Herbaceous & Monsonia & Regular & Regular \\
\hline 5 & Herbaceous & Pelargonium & Irregular & Regular \\
\hline 5 & Succulent & Erodium & Regular & Regular \\
\hline 10 & Succulent & Geranium & Regular & Regular \\
\hline 15 & Herbaceous & Monsonia & Regular & Regular \\
\hline 15 & Succulent & Sarcocaulon & Regular & Regular \\
\hline 15 & Succulent & Sarcocaulon & Regular & Regular \\
\hline 15 & Herbaceous & Monsonia & Regular & Regular \\
\hline 10 & Succulent & Geranium & Regular & Regular \\
\hline 5 & Herbaceous & Erodium & Regular & Regular \\
\hline 5 & Herbaceous & Erodium & Regular & Regular \\
\hline 5 & Herbaceous & Erodium & Regular & Regular \\
\hline 5 & Herbaceous & Erodium & Regular & Regular \\
\hline 12 & Herbaceous & Monsonia & Regular & Regular \\
\hline 12 & Herbaceous & Pelargonium & Irregular & Regular \\
\hline 15 & Succulent & Sarcocaulon & Regular & Regular \\
\hline 10 & Herbaceous & Geranium & Regular & Regular \\
\hline 12 & Herbaceous & Monsonia & Regular & Regular \\
\hline 10 & Herbaceous & Geranium & Regular & Regular \\
\hline 10 & Herbaceous & Geranium & Regular & Regular \\
\hline 5 & Herbaceous & Pelargonium & Irregular & Regular \\
\hline 15 & Herbaceous & Monsonia & Regular & Regular \\
\hline
\end{tabular}

Table 2 Validation results

\begin{tabular}{lllll}
\hline Stamens & Type & Genus & Flowers & Actual \\
\hline 12 & Succulent & Sarcocaulon & Regular & Regular \\
10 & Herbaceous & Pelargonium & Irregular & Regular \\
5 & Succulent & Erodium & Regular & Regular \\
10 & Succulent & Geranium & Regular & Regular \\
5 & Herbaceous & Erodium & Regular & Regular \\
5 & Herbaceous & Pelargonium & Irregular & Regular \\
15 & Succulent & Sarcocaulon & Regular & Regular \\
5 & Succulent & Erodium & Regular & Regular \\
5 & Succulent & Erodium & Regular & Regular \\
12 & Herbaceous & Monsonia & Regular & Regular \\
\hline
\end{tabular}

Table 3 Testing results

\begin{tabular}{lllll}
\hline Stamens & Type & Genus & Flowers & Actual \\
\hline 12 & Succulent & Sarcocaulon & Regular & Regular \\
15 & Succulent & Sarcocaulon & Regular & Regular \\
5 & Succulent & Pelargonium & Irregular & Regular \\
12 & Succulent & Pelargonium & Irregular & Regular \\
10 & Succulent & Geranium & Regular & Regular \\
10 & Herbaceous & Geranium & Regular & Regular \\
12 & Succulent & Sarcocaulon & Regular & Regular \\
5 & Herbaceous & Pelargonium & Irregular & Regular \\
15 & Succulent & Sarcocaulon & Regular & Regular \\
12 & Succulent & Sarcocaulon & Regular & Regular \\
\hline
\end{tabular}

\section{Applications}

Major application of training of neuron is in the neural processor which can be used neural processor that can be in turn used the Robots which acts a Central processing system for analyzing the problem by itself.

\section{Conclusion}

What is claimed in this are

a) Analysis of Biological Neuron was performed on major modules of a Neuron like Resting Membrane Potential, Membrane Time Constant, Membrane length Constant, Axon action potential, Axon voltage clamp, synaptic potential and current and the voltage and current to these neurons are to be considered for a Neural Processor for better results.

b) Training of Neuron is performed and graphs are plotted on neurons can be trained manually and automatically and can be used in Neural Processor.

c) Data Analysis was performed on the Neural Network and results are plotted.

d) Experimental data feed was performed and output results are documented. 
Table 4 Output results for given input for a neuron network

\begin{tabular}{|c|c|c|c|c|c|c|c|}
\hline Species & Frontal lip & Rear width & Length & Width & Depth & Output I & Output II \\
\hline 0 & 20.6 & 14.4 & 42.8 & 46.5 & 19.6 & 0.186163 & 0.702353 \\
\hline I & 13.3 & II.I & 27.8 & 32.3 & 11.3 & 0.149162 & 0.297652 \\
\hline 0 & 16.7 & 14.3 & 32.3 & 37 & 14.7 & 0.212918 & 0.460247 \\
\hline I & 9.8 & 8.9 & 20.4 & 23.9 & 8.8 & 0.261739 & 0.020763 \\
\hline 0 & 15.6 & 14.1 & 31 & 34.5 & 13.8 & 0.202338 & 0.417115 \\
\hline I & 9.1 & 8.1 & 18.5 & 21.6 & 7.7 & 0.294646 & -0.03946 \\
\hline 0 & 14.1 & 10.5 & 29.1 & 31.6 & 13.1 & 0.103071 & 0.448395 \\
\hline I & II.I & 9.9 & 23.8 & 27.1 & 9.8 & 0.206808 & 0.129986 \\
\hline I & 12.8 & 12.2 & 27.9 & 31.9 & 11.5 & 0.189142 & 0.212812 \\
\hline 0 & 19.9 & 16.6 & 39.4 & 43.9 & 17.9 & 0.217477 & 0.493278 \\
\hline 0 & 17.5 & 14.7 & 33.3 & 37.6 & 14.6 & 0.217591 & 0.466217 \\
\hline 0 & 20.1 & 17.2 & 39.8 & 44.1 & 18.6 & 0.220435 & 0.482547 \\
\hline 0 & 19.9 & 17.9 & 40.1 & 46.4 & 17.9 & 0.221478 & 0.474355 \\
\hline I & 21.3 & 15.7 & 47.1 & 54.6 & 20 & 0.188331 & 0.708349 \\
\hline I & 16.4 & 13 & 35.7 & 41.8 & 15.2 & 0.176033 & 0.648427 \\
\hline 0 & 19.7 & 16.7 & 39.9 & 43.6 & 18.2 & 0.216633 & 0.496111 \\
\hline I & 12.8 & 12.2 & 26.7 & 31.1 & II.I & 0.19994 & 0.166002 \\
\hline 0 & 14 & 11.5 & 29.2 & 32.2 & 13.1 & 0.119184 & 0.398798 \\
\hline 0 & 17.4 & 12.8 & 36.1 & 39.5 & 16.2 & $0.174|5|$ & 0.660337 \\
\hline 0 & 10.2 & 8.2 & 20.2 & 22.2 & 9 & 0.134428 & 0.290267 \\
\hline I & 15.7 & 12.6 & 35.8 & 40.3 & 14.5 & 0.174377 & 0.640898 \\
\hline I & 15 & 14.2 & 32.8 & 37.4 & 14 & 0.218203 & $0.442|4|$ \\
\hline 0 & 18.8 & 13.8 & 39.2 & 43.3 & 17.9 & 0.180507 & 0.683173 \\
\hline 0 & 17.6 & 14 & 34 & 38.6 & 15.5 & 0.203173 & 0.516958 \\
\hline 0 & 15.4 & II.I & 30.2 & 33.6 & 13.5 & 0.120094 & 0.485032 \\
\hline I & 11.2 & 10 & 22.8 & 26.9 & 9.4 & 0.229009 & 0.07157 \\
\hline 0 & 18 & 14.9 & 34.7 & 39.5 & 15.7 & 0.217147 & 0.486634 \\
\hline I & 17.1 & 12.6 & 36.4 & 42 & 15.1 & 0.180284 & $0.66967 \mid$ \\
\hline I & 9 & 8.5 & 19.3 & 22.7 & 7.7 & 0.291245 & -0.03931 \\
\hline 0 & 18.9 & 16.7 & 36.3 & 41.7 & 15.3 & 0.221713 & 0.469103 \\
\hline I & 10.4 & 9.7 & 21.7 & 25.4 & 8.3 & 0.264309 & -0.00528 \\
\hline 0 & 13.7 & 11 & 27.5 & 30.5 & 12.2 & 0.117027 & 0.348453 \\
\hline 0 & 19.4 & 14.4 & 39.8 & 44.3 & 17.9 & 0.176706 & 0.665057 \\
\hline I & 16.6 & 13.5 & 38.1 & 43.4 & 14.9 & 0.177909 & 0.660291 \\
\hline I & 12.2 & 10.8 & 27.3 & 31.6 & 10.9 & 0.149458 & 0.275785 \\
\hline I & 15.8 & 15 & 34.5 & 40.3 & 15.3 & 0.218708 & 0.479564 \\
\hline 0 & 14.2 & 10.7 & 27.8 & 30.9 & 12.7 & $0.107 / 55$ & 0.397704 \\
\hline I & 12 & 10.7 & 24.6 & 28.9 & 10.5 & 0.197942 & 0.140803 \\
\hline 0 & 14.3 & 12.2 & 28.1 & 31.8 & 12.5 & 0.152032 & 0.311478 \\
\hline I & 8.1 & 6.7 & 16.1 & 19 & 7 & 0.304009 & -0.02581 \\
\hline I & 13.1 & 10.6 & 28.2 & 32.3 & 11 & 0.134658 & 0.337906 \\
\hline
\end{tabular}




\begin{tabular}{|c|c|c|c|c|c|c|c|}
\hline Species & Frontal lip & Rear width & Length & Width & Depth & Output I & Output II \\
\hline 0 & 13.4 & 10.1 & 26.6 & 29.6 & 12 & 0.100086 & 0.39047 I \\
\hline I & 11.9 & 11.4 & 26 & 30.1 & 10.9 & 0.194433 & 0.153612 \\
\hline 0 & 17.9 & 12.9 & 36.9 & 40.9 & 16.5 & 0.177868 & 0.674719 \\
\hline I & 9.1 & 8.2 & 19.2 & 22.2 & 7.7 & 0.2856 & -0.01869 \\
\hline 0 & 17.1 & 14.5 & 33.1 & 37.2 & 14.6 & 0.21554 & $0.465 \mid 43$ \\
\hline I & 16.2 & 13.3 & 36 & 41.7 & 15.4 & 0.175418 & 0.641807 \\
\hline I & 9.5 & 8.2 & 19.6 & 22.4 & 7.8 & 0.27607 I & 0.003014 \\
\hline 0 & 14.6 & 11.3 & 29.9 & 33.5 & 12.8 & 0.115153 & 0.441021 \\
\hline 0 & 11.4 & 9.2 & 21.7 & 24.1 & 9.7 & 0.137335 & 0.26198 \\
\hline 0 & 13.2 & 11 & 27.1 & 30.4 & 12.2 & 0.116576 & 0.339024 \\
\hline 0 & 17.5 & 12.7 & 34.6 & 38.4 & 16.1 & 0.170864 & 0.637358 \\
\hline 0 & 12.5 & 9.4 & 24.2 & 27 & 11.2 & 0.104548 & 0.363098 \\
\hline 0 & 18.4 & 13.4 & 37.9 & 42.2 & 17.7 & 0.180254 & $0.682|5|$ \\
\hline I & 16.4 & 14 & 34.2 & 39.8 & 15.2 & 0.195791 & $0.5357 \mid 4$ \\
\hline 0 & 15.7 & 12.2 & 31.7 & 34.2 & 14.2 & 0.150165 & 0.50174 \\
\hline 0 & 14.2 & 10.6 & 28.7 & 31.7 & 12.9 & 0.103394 & 0.434736 \\
\hline I & 11.6 & 11.4 & 23.7 & 27.7 & 10 & 0.233572 & 0.044733 \\
\hline I & 19.3 & 13.5 & 41.6 & 47.4 & 17.8 & 0.189698 & 0.709355 \\
\hline I & 9.8 & 8 & 20.3 & 23 & 8.2 & 0.248102 & 0.078089 \\
\hline I & 13.4 & 11.8 & 28.4 & 32.7 & 11.7 & 0.16821 & 0.280037 \\
\hline I & 19.2 & 16.5 & 40.9 & 47.9 & 18.1 & 0.186767 & $0.56508 \mathrm{I}$ \\
\hline I & 15.4 & 13.3 & 32.4 & 37.6 & 13.8 & 0.193727 & 0.484812 \\
\hline I & 17.1 & 12.7 & 36.7 & 41.9 & 15.6 & 0.181442 & 0.674435 \\
\hline 0 & 15.7 & 13.6 & 31 & 34.8 & 13.8 & 0.197068 & 0.41946 \\
\hline I & 17.7 & 13.6 & 38.7 & 44.5 & 16 & 0.18074 & 0.676507 \\
\hline 0 & 15.1 & 11.4 & 30.2 & 33.3 & 14 & 0.123834 & $0.479|4|$ \\
\hline I & 12.8 & 10.2 & 27.2 & 31.8 & 10.9 & 0.13524 & 0.330697 \\
\hline 0 & 12.6 & 11.5 & 25 & 28.1 & 11.5 & 0.14198 & 0.237604 \\
\hline 0 & 23.1 & 15.7 & 47.6 & 52.8 & 21.6 & $0.18868 \mid$ & 0.709758 \\
\hline I & 14.3 & 11.6 & 31.3 & 35.5 & 12.7 & 0.142003 & 0.474487 \\
\hline 0 & 21.9 & 17.2 & 42.6 & 47.4 & 19.5 & 0.205331 & 0.523984 \\
\hline 0 & 16.3 & 11.6 & 31.6 & 34.2 & 14.5 & 0.142465 & $0.54478 \mathrm{I}$ \\
\hline I & 15.2 & 14.3 & 33.9 & 38.5 & 14.7 & 0.210311 & 0.482866 \\
\hline I & 13.1 & 10.9 & 28.3 & 32.4 & 11.2 & 0.139094 & 0.32565 I \\
\hline I & 15.1 & 13.5 & 31.9 & 37 & 13.8 & 0.204531 & 0.448863 \\
\hline 0 & 18.4 & 15.7 & 36.5 & 41.6 & 16.4 & 0.218586 & 0.487638 \\
\hline 0 & 12.9 & 11.2 & 25.8 & 29.1 & 11.9 & 0.134229 & 0.274483 \\
\hline 0 & 18 & 13.4 & 36.7 & 41.3 & 17.1 & 0.17606 & 0.661923 \\
\hline I & 11 & 9.8 & 22.5 & 25.7 & 8.2 & 0.254662 & 0.014069 \\
\hline 1 & 13.9 & 13 & 30 & 34.9 & 13.1 & 0.194687 & $0.356 \mid 25$ \\
\hline 0 & 21.4 & 18 & 41.2 & 46.2 & 18.7 & 0.221432 & 0.475679 \\
\hline 0 & 21 & 15 & 42.9 & 47.2 & 19.4 & 0.180696 & 0.685229 \\
\hline 0 & 18 & 16.3 & 37.9 & 43 & 17.2 & 0.217559 & 0.491183 \\
\hline
\end{tabular}




\begin{tabular}{|c|c|c|c|c|c|c|c|}
\hline Species & Frontal lip & Rear width & Length & Width & Depth & Output I & Output II \\
\hline I & 19.7 & 15.3 & 41.9 & 48.5 & 17.8 & 0.17477 & 0.660315 \\
\hline I & 13 & 11.4 & 27.3 & 31.8 & 11.3 & 0.167924 & 0.24376 \\
\hline 0 & 14.7 & II.I & 29 & 32.1 & 13.1 & 0.114447 & 0.423816 \\
\hline I & 16.8 & 12.8 & 36.2 & 41.8 & 14.9 & 0.17777 & 0.657896 \\
\hline 0 & 18.6 & 13.4 & 37.8 & 41.9 & 17.3 & 0.178853 & 0.676032 \\
\hline 0 & 20.5 & 17.5 & 40 & 45.5 & 19.2 & 0.22045 & 0.481785 \\
\hline I & 12.9 & 11 & 26.8 & 30.9 & 11.4 & 0.160758 & 0.252301 \\
\hline I & 12.8 & 10.9 & 27.4 & 31.5 & 11 & 0.151763 & 0.275038 \\
\hline I & 15 & 11.9 & 32.5 & 37.2 & 13.6 & 0.155423 & 0.552435 \\
\hline I & 10.1 & 9.3 & 20.9 & 24.4 & 8.4 & 0.269591 & -0.00864 \\
\hline I & 10.3 & 9.5 & 21.3 & 24.7 & 8.9 & 0.260331 & 0.009475 \\
\hline I & 16.3 & 12.7 & 35.6 & 40.9 & 14.9 & 0.175943 & 0.648233 \\
\hline I & 13.2 & 12.2 & 27.9 & 32.1 & 11.5 & 0.189134 & 0.22384 \\
\hline 0 & 18.6 & 14.5 & 34.7 & 39.4 & 15 & 0.215057 & 0.492643 \\
\hline I & 19.8 & 14.2 & 43.2 & 49.7 & 18.6 & 0.189354 & 0.709639 \\
\hline I & 9.6 & 7.9 & 20.1 & 23.1 & 8.2 & 0.247557 & $0.08367 \mid$ \\
\hline 0 & 23.1 & 20.2 & 46.2 & 52.5 & 21.1 & 0.221067 & 0.473831 \\
\hline 0 & 18.8 & 13.4 & 37.2 & 41.1 & 17.5 & 0.177726 & 0.6683 \\
\hline 0 & 14.1 & 10.7 & 28.7 & 31.9 & 13.3 & 0.104427 & 0.439446 \\
\hline I & 11.5 & 11 & 24.7 & 29.2 & 10.1 & 0.211085 & 0.105349 \\
\hline 0 & 19.4 & I4.I & 39.1 & 43.2 & 17.8 & 0.177216 & 0.666064 \\
\hline 0 & 16.2 & 11.8 & 32.3 & 35.3 & 14.7 & 0.147442 & 0.567963 \\
\hline 0 & 14.2 & 11.3 & 29.2 & 32.2 & 13.5 & 0.114583 & 0.425135 \\
\hline I & 12.6 & 10 & 27.7 & 31.7 & 11.4 & 0.131482 & 0.363198 \\
\hline I & 11.8 & 9.6 & 24.2 & 27.8 & 9.7 & 0.183267 & 0.19081 \\
\hline 0 & 20.3 & 16 & 39.4 & 44.1 & 18 & 0.205989 & 0.52638 \\
\hline I & 17.9 & 14.1 & 39.7 & 44.6 & 16.8 & 0.179703 & 0.674052 \\
\hline 0 & 23 & 16.8 & 47.2 & 52.1 & 21.5 & 0.177519 & 0.676243 \\
\hline I & 17.2 & 13.5 & 37.6 & 43.9 & 16.1 & 0.179544 & 0.670033 \\
\hline I & 14.6 & 11.3 & 31.9 & 36.4 & 13.7 & 0.146795 & 0.537008 \\
\hline I & 15.2 & 12.1 & 32.3 & 36.7 & 13.6 & 0.158385 & 0.539258 \\
\hline I & 16.9 & 13.2 & 37.3 & 42.7 & 15.6 & 0.179661 & 0.667931 \\
\hline I & 15.5 & 13.8 & 33.4 & 38.7 & 14.7 & 0.197028 & 0.511104 \\
\hline 0 & 18.5 & 14.6 & 37 & 42 & 16.6 & 0.187866 & 0.575208 \\
\hline 0 & 14.7 & 13.2 & 29.6 & 33.4 & 12.9 & 0.176516 & 0.354553 \\
\hline I & 15.9 & 12.7 & 34 & 38.9 & 14.2 & 0.170094 & 0.594635 \\
\hline I & 15 & 13.8 & 31.7 & 36.9 & 14 & 0.213529 & 0.433439 \\
\hline 0 & 18.3 & 15.7 & 35.1 & 40.5 & 16.1 & 0.220926 & 0.47552 \\
\hline 0 & I5.I & 11.5 & 30.9 & 34 & 13.9 & 0.126107 & 0.496218 \\
\hline I & 13.7 & 12.5 & 28.6 & 33.8 & 11.9 & 0.189198 & 0.278252 \\
\hline 0 & 19.1 & 16.3 & 37.9 & 42.6 & 17.2 & 0.219603 & 0.485798 \\
\hline 0 & 20.6 & 17.5 & 41.5 & 46.2 & 19.2 & 0.216969 & 0.49303 \\
\hline 0 & 21.3 & 18.4 & 43.8 & 48.4 & 20 & 0.217212 & 0.490055 \\
\hline
\end{tabular}




\begin{tabular}{|c|c|c|c|c|c|c|c|}
\hline Species & Frontal lip & Rear width & Length & Width & Depth & Output I & Output II \\
\hline 0 & 10.7 & 8.6 & 20.7 & 22.7 & 9.2 & 0.138393 & 0.270389 \\
\hline 0 & 17.5 & 12 & 34.4 & 37.3 & 15.3 & 0.167035 & 0.639669 \\
\hline 1 & 13.1 & 11.5 & 27.6 & 32.6 & 11.1 & 0.166793 & 0.251855 \\
\hline I & 18.8 & 15.8 & 42.1 & 49 & 17.8 & 0.172055 & 0.643966 \\
\hline 0 & 19.1 & 16 & 37.8 & 42.3 & 16.8 & 0.218212 & 0.490693 \\
\hline 0 & 12.7 & 10.4 & 26 & 28.8 & 12.1 & 0.108675 & 0.349789 \\
\hline 0 & 17.5 & 14.3 & 34.5 & 39.6 & 15.6 & 0.204839 & 0.516065 \\
\hline 0 & 14 & 11.9 & 27 & 31.4 & 12.6 & 0.145336 & 0.297399 \\
\hline 0 & 20 & 16.7 & 40.4 & 45.1 & 17.7 & 0.214122 & 0.501655 \\
\hline I & 15.7 & 13.9 & 33.6 & 38.5 & 14.1 & 0.205099 & 0.490191 \\
\hline 0 & 18.6 & 13.5 & 36.9 & 40.2 & 17 & 0.175904 & 0.649831 \\
\hline I & 14.7 & 12.5 & 30.1 & 34.7 & 12.5 & 0.184551 & $0.374 I 76$ \\
\hline 0 & 16.1 & 13.7 & 31.4 & 36.1 & 13.9 & 0.201979 & 0.439438 \\
\hline 0 & 16.2 & 14 & 31.6 & 35.6 & 13.7 & 0.206085 & $0.43467 I$ \\
\hline 0 & 18.4 & 15.5 & 35.6 & 40 & 15.9 & 0.220283 & 0.479205 \\
\hline I & 19.3 & 13.8 & 40.9 & 46.5 & 16.8 & 0.184746 & 0.694301 \\
\hline I & 19.8 & 14.3 & 42.4 & 48.9 & 18.3 & 0.186874 & 0.702543 \\
\hline I & 15 & 11.5 & 32.4 & 37 & 13.4 & 0.151531 & 0.551016 \\
\hline 0 & 10.7 & 9.7 & 21.4 & 24 & 9.8 & 0.148883 & 0.221458 \\
\hline I & 7.2 & 6.5 & 14.7 & 17.1 & 6.1 & 0.334492 & -0.09989 \\
\hline 0 & 21.5 & 15.5 & 45.5 & 49.7 & 20.9 & 0.185484 & 0.700857 \\
\hline 0 & 17.5 & 14.4 & 34.5 & 39 & 16 & 0.207533 & 0.51132 \\
\hline 0 & 17.1 & 12.6 & 35 & 38.9 & 15.7 & 0.169674 & 0.643116 \\
\hline 0 & 18.8 & 15.2 & 35.8 & 40.5 & 16.6 & 0.216638 & 0.495743 \\
\hline I & 17.4 & 16.9 & 38.2 & 44.1 & 16.6 & 0.222903 & 0.474585 \\
\hline 0 & 12.5 & 9.4 & 23.2 & 26 & 10.8 & 0.117397 & 0.31905 \\
\hline I & 17.5 & 16.7 & 38.6 & 44.5 & 17 & 0.21872 & 0.487265 \\
\hline 0 & 21.7 & I7.I & 41.7 & 47.2 & 19.6 & 0.207684 & 0.518693 \\
\hline 0 & 20.9 & 16.5 & 39.9 & 44.7 & 17.5 & 0.216313 & 0.49661 \\
\hline I & 12.6 & 12.2 & 26.1 & 31.6 & 11.2 & 0.1997 & 0.160681 \\
\hline 0 & 16.1 & 13.6 & 31.6 & 36 & 14 & 0.20021 & 0.445052 \\
\hline 0 & 14 & 12.8 & 28.8 & 32.4 & 12.7 & 0.161376 & 0.317749 \\
\hline I & 15.6 & 14.7 & 33.9 & 39.5 & 14.3 & 0.220551 & 0.464055 \\
\hline I & 18 & 13.7 & 39.2 & 44.4 & 16.2 & 0.180954 & $0.67794 \mid$ \\
\hline 0 & 21.9 & 15.7 & 45.4 & 51 & 21.1 & 0.18446 & 0.698053 \\
\hline 0 & 12.5 & 10 & 24.1 & 27 & 10.9 & 0.12394 & $0.29487 \mid$ \\
\hline I & 9.2 & 7.8 & 19 & 22.4 & 7.7 & 0.271036 & 0.025815 \\
\hline I & 11.6 & 11 & 24.6 & 28.5 & 10.4 & 0.2124 & 0.103078 \\
\hline I & 15.6 & 13.9 & 32.8 & 37.9 & 13.4 & 0.214488 & 0.451173 \\
\hline 0 & 9.1 & 6.9 & 16.7 & 18.6 & 7.4 & 0.168183 & 0.230079 \\
\hline 0 & 21.6 & 15.4 & 45.7 & 49.7 & 20.6 & 0.185995 & 0.702309 \\
\hline I & 13.9 & II.I & 29.2 & 33.3 & 12.1 & 0.135032 & 0.38883 \\
\hline I & 11.7 & 10.6 & 24.9 & 28.5 & 10.4 & 0.197175 & 0.1446 \\
\hline
\end{tabular}




\begin{tabular}{|c|c|c|c|c|c|c|c|}
\hline Species & Frontal lip & Rear width & Length & Width & Depth & Output I & Output I \\
\hline 0 & 11.4 & 9 & 22.7 & 24.8 & 10.1 & 0.117034 & 0.325353 \\
\hline I & 15.4 & 11.8 & 33 & 37.5 & 13.6 & 0.158811 & 0.574402 \\
\hline I & 14.9 & 13.2 & 30.1 & 35.6 & 12 & 0.208719 & 0.352757 \\
\hline I & 10.8 & 9 & 23 & 26.5 & 9.8 & 0.191608 & 0.191765 \\
\hline I & 15.1 & 13.8 & 31.7 & 36.6 & 13 & 0.21727 & 0.409333 \\
\hline 0 & 21.6 & 14.8 & 43.4 & 48.2 & 20.1 & 0.185089 & 0.699409 \\
\hline I & 11.6 & 9.1 & 24.5 & 28.4 & 10.4 & 0.159586 & 0.279538 \\
\hline 0 & 14.1 & 10.4 & 28.9 & 31.8 & 13.5 & 0.103647 & 0.457285 \\
\hline 0 & 17.8 & 12.5 & 36 & 39.8 & 16.7 & 0.177742 & 0.67555 \\
\hline I & 16.7 & 16.1 & 36.6 & 41.9 & 15.4 & 0.224569 & 0.469772 \\
\hline I & 10.8 & 9.5 & 22.5 & 26.3 & 9.1 & $0.227 \mid 26$ & $0.08676 \mathrm{I}$ \\
\hline I & I5.I & 13.3 & 31.8 & 36.3 & 13.5 & 0.201555 & 0.43998 \\
\hline I & 15 & 10.9 & 31.4 & 36.4 & 13.2 & 0.14299 & 0.528109 \\
\hline I & 16.1 & 11.6 & 33.8 & 39 & 14.4 & 0.169483 & 0.628457 \\
\hline I & 15.3 & 14.2 & 32.6 & 38.3 & 13.8 & 0.218605 & 0.445497 \\
\hline 0 & 15 & 12.3 & 30.1 & 33.3 & 14 & 0.15382 & 0.421494 \\
\hline I & 12.8 & 11.7 & 27.1 & 31.2 & 11.9 & 0.178291 & 0.223974 \\
\hline I & 16.1 & 12.8 & 34.9 & 40.7 & 15.7 & 0.175651 & 0.64623 \\
\hline 0 & 14.9 & 13 & 30 & 33.7 & 13.3 & 0.176118 & $0.37604 I$ \\
\hline I & 11.8 & 10.5 & 25.2 & 29.3 & 10.3 & 0.184681 & $0.1756 \mid 4$ \\
\hline 0 & 15.6 & 13.5 & 31.2 & 35.1 & 14.1 & 0.195013 & 0.429867 \\
\hline 0 & 18.2 & 13.7 & 38.8 & 42.7 & 17.2 & 0.178856 & 0.677047 \\
\hline 0 & 20.1 & 13.7 & 40.6 & 44.5 & 18 & 0.184155 & 0.695958 \\
\hline 0 & 22.1 & 15.8 & 44.6 & 49.6 & 20.5 & 0.179218 & 0.680919 \\
\hline 0 & 22.5 & 17.2 & 43 & 48.7 & 19.8 & 0.198351 & 0.540444 \\
\hline I & 12.3 & II & 26.8 & 31.5 & II. 4 & 0.155959 & 0.259127 \\
\hline I & 12 & II.I & 25.4 & 29.2 & 11 & 0.194869 & 0.151061 \\
\hline I & 8.8 & 7.7 & 18.1 & 20.8 & 7.4 & 0.296807 & -0.03373 \\
\hline I & 16.2 & 15.2 & 34.5 & 40.1 & 13.9 & 0.22636 & 0.455678 \\
\hline 0 & 15.6 & 14 & 31.6 & 35.3 & 13.8 & 0.202609 & 0.426803 \\
\hline
\end{tabular}

\section{Acknowledgments}

Author would like to thank Prof. Navarun Gupta, Prof. Hassan Bajwa and Prof. Linfeng Zhang for their academic support. Author also thanks anonymous reviewers for their comments.

\section{Conflicts of interest}

The author declares there are no conflicts of interest.

\section{References}

1. George Vastianos. Artificial Biomorphic Neurocontrolled Insect.

2. Aaron Saenz. Amazing Robot Controlled By Rat Brain Continues Progress. 2010.
3. Zarmeen Shahzad. Possibility That Scientists Might Have Discovered The Building Blocks Of Our Memory. 2016.

4. Neural Networks in R tutorial.

5. Newman MH, Newman EA. MetaNeuron: A free neuron simulation program for teaching cellular neurophysiology. $J$ Undergrad Neurosci Educ. 2013;12(1):A11-A7.

6. Net Maker.

7. Jaden Tool. 